\title{
Alveolar bone thickness and fenestration of incisors in untreated Korean patients with skeletal class III malocclusion: A retrospective 3-dimensional cone-beam computed tomography study
}

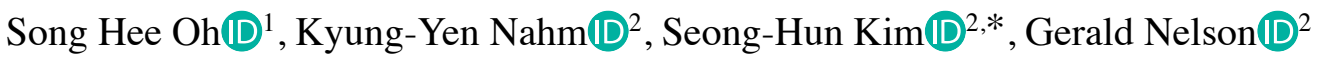 \\ ${ }^{1}$ Department of Oral and Maxillofacial Radiology, Graduate School, Kyung Hee University, Seoul, Korea \\ ${ }^{2}$ Department of Orthodontics, Graduate School, Kyung Hee University, Seoul, Korea
}

\section{ABSTRACT}

\begin{abstract}
Purpose: The purpose of this study was to evaluate vertical bone loss and alveolar bone thickness in the maxillary and mandibular incisors of patients with skeletal class III malocclusion. This study also aimed to evaluate the periodontal condition of class III malocclusion patients who had not undergone orthodontic treatment.

Materials and Methods: The sample included cone-beam computed tomography scans of 24 Korean subjects ( 3 male and 21 female). Alveolar bone thickness (ABT), alveolar bone area (ABA), alveolar bone loss (ABL), and fenestration of the maxillary and mandibular incisors were measured using 3-dimensional imaging software.

Results: All incisors displayed an ABT of less than $1.0 \mathrm{~mm}$ from the labial surface to root level 7 (70\% of the root length). A statistically significant difference was observed between the mandibular labial and lingual ABAs and between the maxillary labial and mandibular labial ABAs. The lingual ABA of the mandibular lateral incisors was larger than that of the mandibular central incisors. ABL was severe on the labial surface. A statistically significant difference was observed between the maxillary and mandibular labial ABL values ( $21.8 \%$ and $34.4 \%$, respectively). Mandibular lingual ABL $(27.6 \%)$ was significantly more severe than maxillary lingual ABL $(18.3 \%)(P<0.05)$. Eighty-two fenestrations were found on the labial surfaces of the incisors, while only 2 fenestrations were observed on the lingual surfaces. Fenestrations were most commonly observed at root level 6.

Conclusion: Careful evaluation is needed before orthodontic treatment to avoid iatrogenic damage of periodontal support when treating patients with class III malocclusion. (Imaging Sci Dent 2020; 50: 9-14)
\end{abstract}

KEY WORDS: Alveolar Process; Incisor; Tooth Supporting Structures

\section{Introduction}

Orthodontic treatment can produce excellent aesthetic facial and dental outcomes, but periodontal complications, such as vertical bone loss, bone dehiscence, fenestration, and gingival recession, may occur. ${ }^{1}$ These periodontal problems are particularly common in older patients, especially in the mandibular anterior teeth.

Patients with skeletal class III malocclusion are treated with orthodontic camouflage or surgery-assisted ortho-

\footnotetext{
*Song Hee Oh and Kyung-Yen Nahm contributed equally as co-first authors of this manuscript.

Received September 24, 2019; Revised January 15, 2020; Accepted January 25, 2020 *Correspondence to : Prof. Seong-Hun Kim

Department of Orthodontics, Graduate School, Kyung Hee University, \#1 Hoegidong, Dongdaemun-gu, Seoul 02447, Korea

Tel) 82-2-958-9392,E-mail) bravortho@khu.ac.kr
}

dontics. During orthodontic treatment, the incisors may be moved labio-lingually for dental compensation or decompensation. At that time, the thickness of the alveolar bone supporting the anterior teeth is an important consideration in determining the amount of labio-lingual movement of the tooth. ${ }^{2}$ According to several studies, ${ }^{3,4}$ some compensatory bone formation can be expected to accompany anterior tooth movement; however, in adults, the labial alveolar bone may be very thin or absent.

Other studies have documented patients' pre-treatment periodontal status and alveolar bone thickness. ${ }^{5,6}$ These studies have concluded that even before orthodontic treatment begins, incisal periodontal support may be poor, and severe alveolar bone loss may have occurred. The orthodontist needs to avoid causing further loss of the periodontal support around the incisors, especially in the mandibular

Copyright (c) 2020 by Korean Academy of Oral and Maxillofacial Radiology

This is an Open Access article distributed under the terms of the Creative Commons Attribution Non-Commercial License (http://creativecommons.org/licenses/by-nc/3.0) which permits unrestricted non-commercial use, distribution, and reproduction in any medium, provided the original work is properly cited. Imaging Science in Dentistry · pISSN 2233-7822 eISSN 2233-7830 
lingual bone plate area.

Particularly in adults, orthodontic treatment planning must include a careful clinical and radiographic evaluation for identification of periodontal problems such as dehiscence, fenestrations, or alveolar bone loss before initiating treatment. The goal is to document any periodontal problems and to structure the treatment to preserve or improve, and avoid aggravating, periodontal deficiencies. ${ }^{7}$

The alveolar bone has traditionally been evaluated with standard dental radiography, such as periapical or bitewing radiographs. However, because of the diverse angles of the $\mathrm{X}$-ray source, these images are difficult to standardize, and image distortions are inevitable. The vector of the radiation beam does not allow assessment of alveolar bone changes, such as dehiscence, on the labio-lingual side. ${ }^{8}$ Lateral cephalograms have also been used to evaluate the anterior teeth and the alveolar bone of the anterior palate and symphysis. ${ }^{9}$ However, in these 2-dimensional images, identification errors can occur regarding the anatomical structure to be measured, which degrades measurement accuracy. Three-dimensional (3D) cone-beam computed tomography (CBCT) is one of the best imaging tools available for evaluating bone serving as periodontal support in specific areas of interest. ${ }^{10}$

This study used the pretreatment CBCT records of Korean patients with untreated skeletal class III malocclusion. Its purposes were to measure alveolar bone thickness (ABT), alveolar bone area (ABA), and alveolar bone loss $(\mathrm{ABL})$; to assess the number of fenestrations; and to compare the results between the upper and lower incisors, the central and lateral incisors, and the labial and lingual sides.

\section{Materials and Methods}

\section{Patients}

The experiment draft and its related data were reviewed and approved by the Institutional Review Board of our institution (CUMC11U045). Twenty-four patients with skel- etal class III malocclusion ( 3 male and 21 female; mean age, 22.6 years), who were treated at the Department of Orthodontics at Kyung Hee University Dental Hospital in Seoul, Korea, were recruited for the present study. The patients had no craniofacial anomalies. The exclusion criteria included severe dental crowding, radiological signs of periodontal disease, history of root canal treatment, and rotated teeth.

Pre-treatment lateral cephalograms and 3D CBCT scans were taken at the Department of Oral and Maxillofacial Radiology at our institution. To evaluate the relationships between each patient's bones and teeth, lateral cephalograms were obtained at a peak kilovoltage of $78 \mathrm{kVp}$, a current of $20 \mathrm{~mA}$, and a scan time of 0.7 seconds. CBCT scans (Alphard-Vega, Asahi Roentgen Ind. Co., Ltd, Kyoto, Japan) were obtained for each patient with a field of view (FOV) of $200 \mathrm{~mm} \times 179 \mathrm{~mm}$, a peak kilovoltage of $80 \mathrm{kVp}$, a current of $10 \mathrm{~mA}$, and a scan time of 17 seconds. CBCT datasets were stored in Digital Imaging Communications in Medicine format and were reorganized into 3D images using a multiplanar reformation software program (OnDemand 3D, Cybermed Co., Seoul, Korea). Sagittal sections were created to show 192 labial and 192 lingual surfaces from 4 maxillary incisors and 4 mandibular incisors in the 24 patients. The sagittal sections of the 4 longest maxillary and mandibular incisors were evaluated on the labial and lingual aspects on the horizontal plane.

\section{Measurements}

All multiplanar reformation and CBCT images were measured using the same computer and 24-inch display monitor at a frequency of $60 \mathrm{~Hz}$ and a resolution of $1920 \times 1440$ pixels. Sagittal images of the incisors, in which the image section passed through the midpoint of the incisor section from the root apex, were obtained with the 3D imaging software (OnDemand 3D). Table 1 lists the reference points, lines, and variables used in the present study, and Fig. 1 provides a schematic diagram. Two orthodontists took the alveolar bone measurements, and all

Table 1. Definitions of the measurements used in this study

\begin{tabular}{ll}
\hline Measurement variable & \multicolumn{1}{c}{ Definition } \\
\hline UABT & Upper labial or lingual distance from the root surface to the cortical bone at each root level $(0$ to 10$)$ \\
LABT & Lower labial or lingual distance from the root surface to the cortical bone at each root level $(0$ to 10$)$ \\
$\mathrm{ABA}\left(\mathrm{mm}^{2}\right)$ & Labial or lingual alveolar bone area: area of the small trapezoid between root levels on the cross-section \\
$\mathrm{ABL}(\%)$ & Percentage of root length that displays labial or lingual bone loss \\
Fenestration & Number of tooth fenestrations \\
\hline
\end{tabular}

UABT: upper alveolar bone thickness, LABT: lower alveolar bone thickness, ABA: alveolar bone area, ABL: alveolar bone loss 
CBCT images were measured under dark indoor lighting conditions.

ABT, the distance from the root surface to the cortical bone perpendicular to the root axis, was recorded for every one-tenth of the root length on the labial and lingual aspects (level 0, cementoenamel junction [CEJ] area; level 10, root apex area). The ABA between root levels was assumed to be trapezoidal. The ABA of each root level was calculated as $\frac{1}{2}(a+b)\left(\frac{1}{10}\right.$ of the root length). ABL was defined as the ratio of the distance between the CEJ and the alveolar crestal bone to the root length. Fenestrations were defined as localized defects in the alveolar bone which exposed the root surface without involving the alveolar margin.

The location of all teeth was described pursuant to FDI World Dental Federation international 2-digit notation.

\section{Statistical analysis}

SAS/STAT ${ }^{\circledR}$ software (SAS Institute Inc., Cary, NC, USA) was used for analyses. Intraoperator measurement error was assessed by randomly selecting 8 of the 24 patient datasets and having the same orthodontist measure the values again at a 2 -week interval to exclude the learning effect. Mean, standard deviation, and frequency were used as variables. Interoperator variability was evaluated by analyzing the Pearson correlation coefficient of the additional measurement of the randomly-selected 8 patients by the

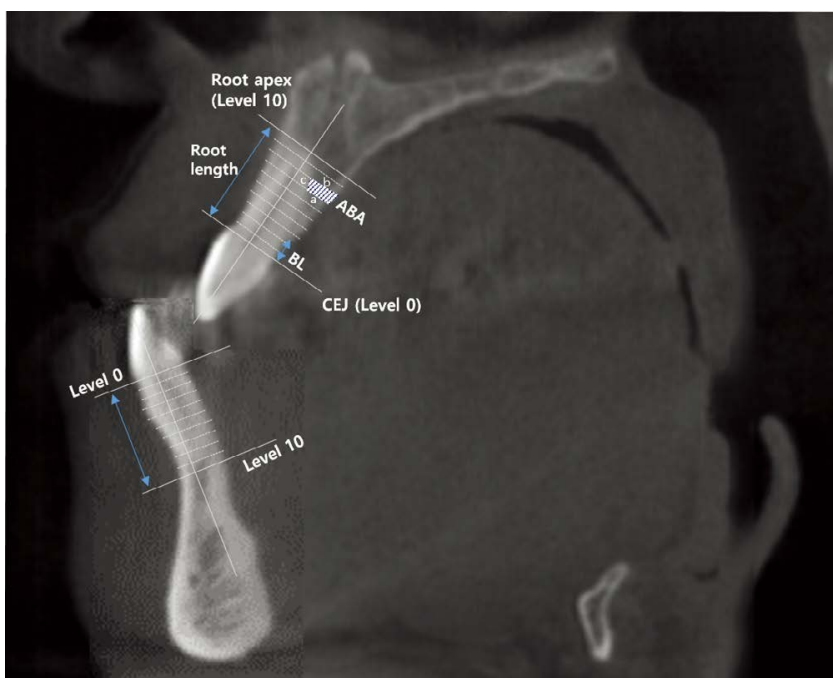

Fig. 1. A schematic diagram of the maxillary and mandibular reference points, lines, and measurement variables used in the present study. ABA: alveolar bone area $\left(\mathrm{mm}^{2}\right)=\left[\frac{1}{2}(\mathrm{a}+\mathrm{b}) \times \mathrm{c}\right]$, level 0 : line perpendicular to the root axis in the cementoenamel junction (CEJ) area, level 10: line perpendicular to the root axis in the root apex area, BL: bone loss. other orthodontist, also 2 weeks after initial measurement. The Student $t$-test, assuming a normal distribution and a 5\% significance level, was used to compare the means of the subgroups.

\section{Results}

Table 2 details a cephalometric analysis of the study subjects. This study confirmed a very high level of interobserver reliability for all variables, with a Pearson correlation coefficient of 0.97 .

\section{ABT}

As shown in Fig. 2, all incisors showed less than $1.0 \mathrm{~mm}$ of ABT on the labial surface up to root level 7. The palatal and lingual bone was generally thicker than the labial bone. The ABT values of the labial and lingual aspects were similar up to root level 3, but beyond that point to root level 10 , the difference was more substantial. The maximum ABT was $6.9 \mathrm{~mm}$ for the left upper central incisor on the palatal side. As with the shape of the labial alveolar bone, the graph of the upper and lower ABT took the shape of an S-curve. In other words, ABT tends to have a smaller value at the middle of the root length than at the coronal third, especially in the lower incisors. The graph of the lingual ABT resembles a linear function.

\section{ABA}

The mean area of each tooth is shown in Fig. 3. A statistically significant difference was observed between the areas of the upper and lower incisors $(P<0.05)$. The mean area of bone above the maxillary incisors was $18.6 \mathrm{~mm}^{2}$

Table 2. Cephalometric characteristics of the samples

\begin{tabular}{lcc}
\hline \multicolumn{1}{c}{ Measurement } & Mean \pm SD & Range \\
\hline SNA & $81.5 \pm 2.7$ & $75.0-86.1$ \\
SNB & $81.0 \pm 2.4$ & $76.6-86.4$ \\
ANB difference & $0.4 \pm 1.8$ & $-3.9-3.6$ \\
FMA & $26.6 \pm 4.8$ & $18.1-37.3$ \\
FMIA & $60.4 \pm 5.1$ & $51.3-72.9$ \\
IMPA & $93.0 \pm 5.4$ & $81.3-104.2$ \\
U1 to FH & $119.8 \pm 4.8$ & $111.1-126.3$ \\
U1 to SN & $111.1 \pm 4.7$ & $101.7-119.7$ \\
Interincisal angle & $120.6 \pm 6.9$ & $111.1-132.6$ \\
\hline
\end{tabular}

SNA: sella-nasion to A point angle, SNB: sella-nasion to B point angle, ANB: A point to B point angle, FMA: Frankfort mandibular plane angle, FMIA: Frankfort mandibular incisor angle, IMPA: incisor mandibular plane angle, U1: upper incisor, FH: Frankfort horizontal line, SN: sellanasion line 

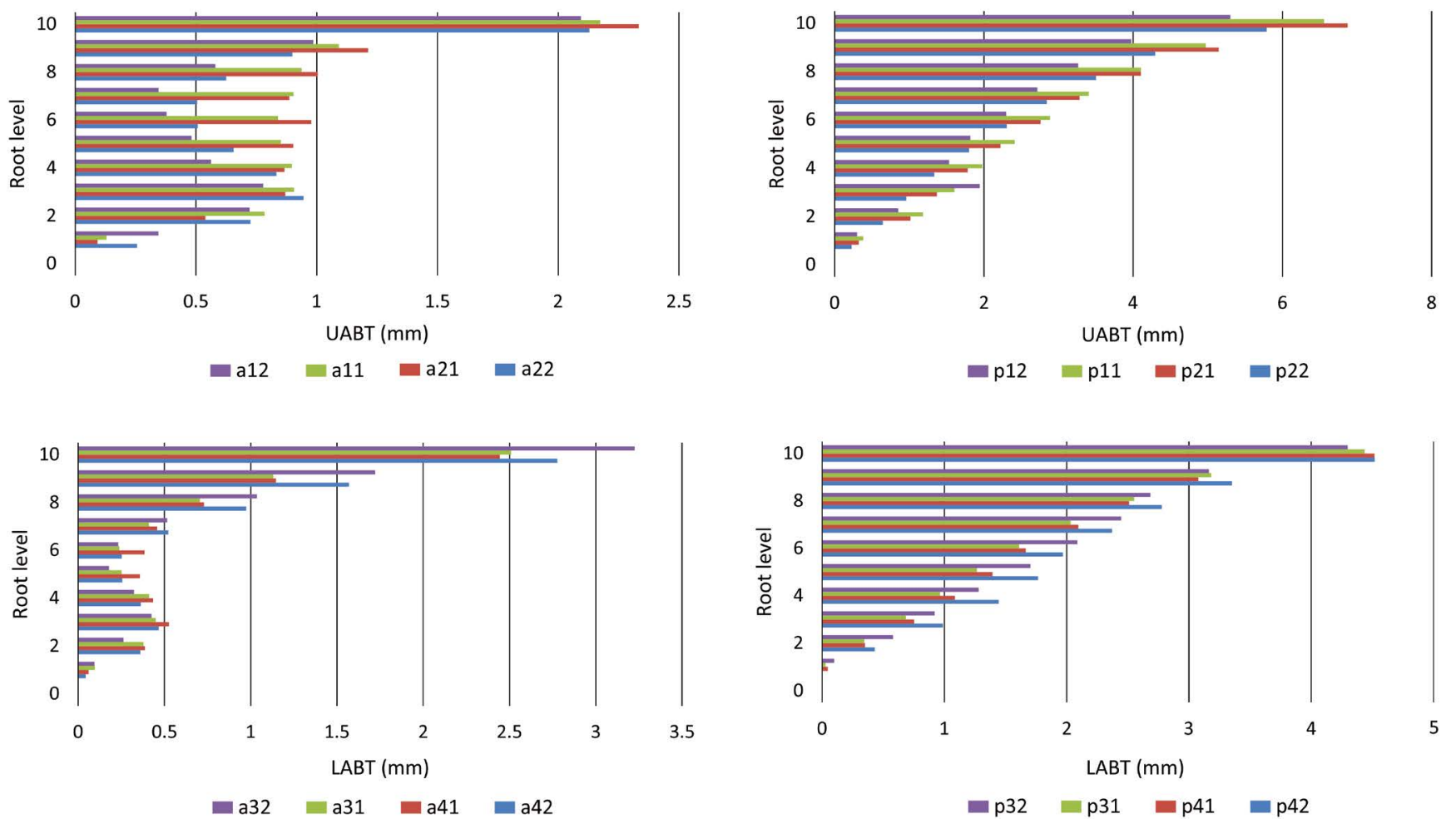

Fig. 2. Alveolar bone thickness (ABT) at each root level (0-10) for each of the incisors (\#12-22, \#32-42). a: labial side, p: lingual side.

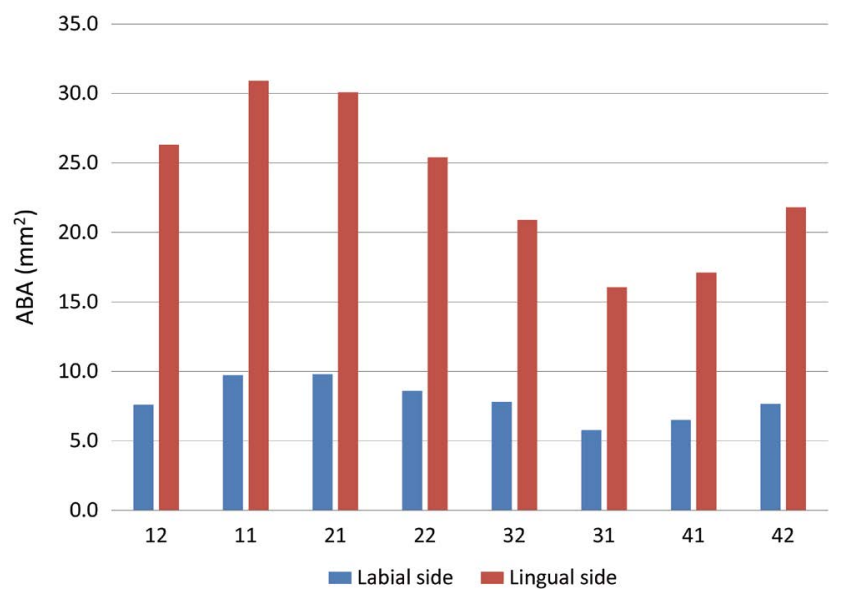

Fig. 3. Alveolar bone area $\left(\mathrm{ABA}, \mathrm{mm}^{2}\right)$ of incisor cross-sections. A statistically significant difference was observed between the upper and lower incisors.

(range, 7.6-30.9 $\mathrm{mm}^{2}$ ), while the mean lower incisor bone area was $13.0 \mathrm{~mm}^{2}$ (range, $5.8-21.8 \mathrm{~mm}^{2}$ ). The mean area of the mandibular lingual cross-section was about 3 times greater than the mean labial area (lingual area, $19.0 \mathrm{~mm}^{2}$; labial area, $6.9 \mathrm{~mm}^{2}$ ). The most significant difference was noted between the mandibular labial and lingual areas $(P<0.05)$. The upper labial cross-sectional area was larger than the lower labial area (upper area, $8.9 \mathrm{~mm}^{2}$; lower area, $\left.6.9 \mathrm{~mm}^{2}\right)$. The labial areas of the maxillary and mandibular incisors were also significantly different $(P<0.05)$. With respect to the central and lateral incisors, the maxillary central and mandibular lateral incisors had greater areas than the maxillary lateral and mandibular central incisors, respectively. However, the only statistically significant difference observed regarding the central and lateral incisors was between the lingual areas of the lower central incisors and the lower lateral incisors $(P<0.05)$.

\section{$A B L$}

ABL was determined by the ratio of the distance between the CEJ and the alveolar crestal bone to the root length. As shown in Figure 4, general ABL was more severe at the labial aspect than the lingual aspect, except in the upper right lateral incisor. $\mathrm{ABL}$ also appeared to be more pronounced in the mandible than in the maxilla. The most severe ABL was on the labial aspect of the lower left lateral incisor $(37.5 \%)$. The lingual aspect of the upper right central incisor had the least ABL (16.3\%). Statistically significant differences were found between the upper labial ABL (21.8\%) and the lower labial ABL (34.4\%) $(P=0.004)$. The lower lingual $\mathrm{ABL}(27.6 \%)$ was significantly more severe than the upper lingual ABL $(18.3 \%)(P<0.05)$. 


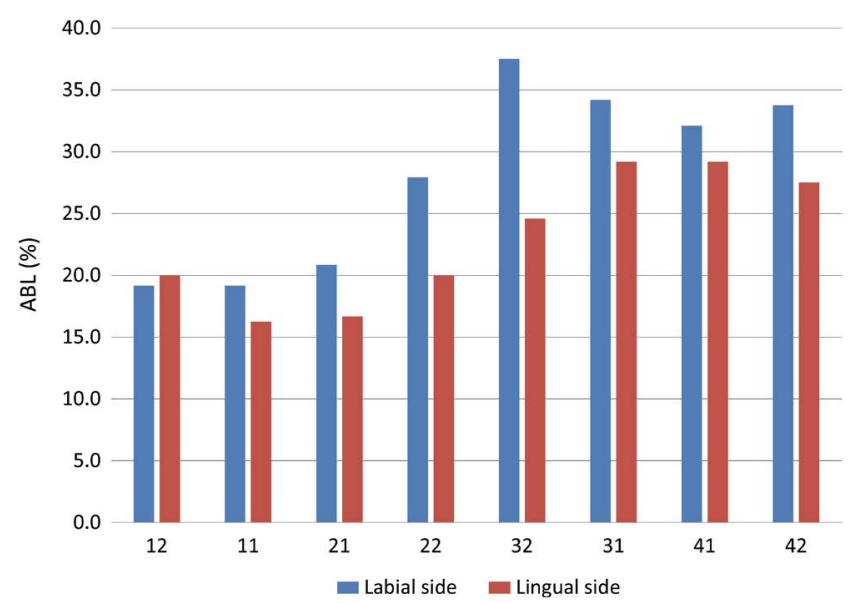

Fig. 4. Alveolar bone loss (ABL) of the labial and lingual sides (\%).

Table 3. Distribution of fenestrations

\begin{tabular}{lrrrrrrrr}
\hline & 12 & 11 & 21 & 22 & 32 & 31 & 41 & 42 \\
\hline Labial side & 16 & 1 & 3 & 10 & 13 & 13 & 10 & 16 \\
Lingual side & 0 & 0 & 0 & 1 & 1 & 0 & 0 & 0 \\
\hline
\end{tabular}

\section{Fenestration}

Fenestration was confirmed in 84 of 192 teeth. Of these, the fenestrations on the left upper and lower lateral incisors were on the lingual side, while the rest were on the labial side. Fenestration was confirmed in 31 maxillary teeth, and the remaining 52 teeth in which fenestrations were observed were mandibular. The distribution of alveolar fenestrations is shown in Table 3. In particular, fenestration tended to occur more frequently in the maxillary incisors. The location of each fenestration was indicated by the root level, as shown in Figure 5. The most prevalent location was root level 6 .

\section{Discussion}

In this study, palatal and lingual bone was generally thicker than labial bone. All incisors showed $<1.0 \mathrm{~mm}$ of ABT up to root level 7 on the labial surface. Lee et al. ${ }^{11}$ previously evaluated the maxillary anterior buccal bone of Korean adults using CBCT. The results resembled those of the present study in that the palatal bone was thicker than the labial bone in the anterior maxilla, although the anterior buccal alveolar bone thickness at the root apex was remarkably thinner than in our study. This may be explained by the inclination of the upper incisors, because the samples of the previous study were not categorized by the Angle clas-

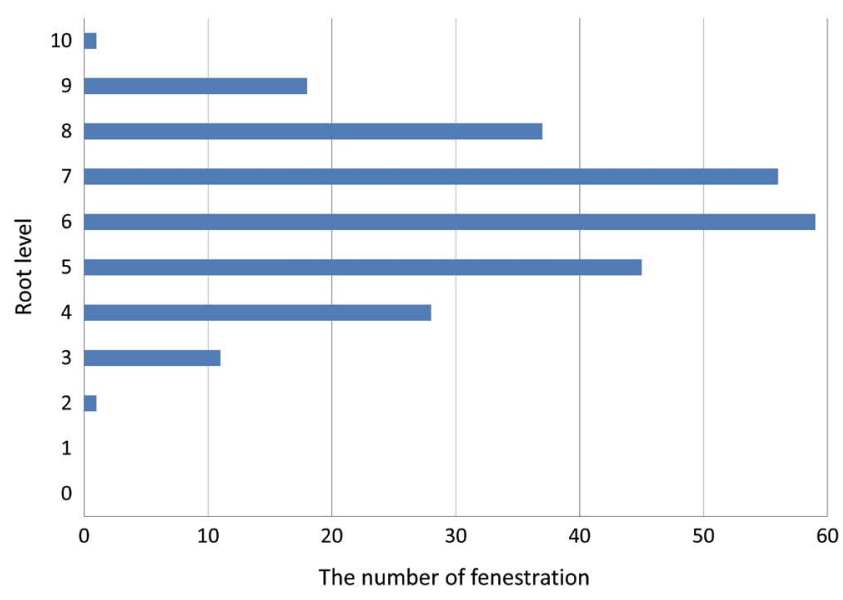

Fig. 5. Prevalence of fenestrations according to root level.

sification.

$\mathrm{ABL}$ was more severe on the labial than the lingual side, with the exception of the right upper lateral incisor. ABL also appeared to be more pronounced in the mandible than in the maxilla. Interestingly, a previous study analyzed class I patients with bi-dentoalveolar protrusion and showed the opposite results: the lower labial region had an ABL of $27.0 \%$, while the ABL of the lower lingual plate was $31.3 \%$. $^{6}$ This difference could be explained by the compensatory inclination of the anterior teeth to accommodate the skeletal discrepancy in class III patients.

Fenestration was confirmed in 84 out of 192 teeth. Of these, only fenestrations of the left upper and lower lateral incisors were found on the lingual aspect, with the rest on the labial aspect. Yagci et al. ${ }^{12}$ evaluated fenestration and dehiscence in skeletal malocclusions using CBCT. These alveolar defects were prominent on the buccal root surface; the class III group also showed more alveolar defects in the mandible than the maxilla ( $45.0 \%$ vs. $29.4 \%$, respectively). Evangelista et al. ${ }^{13}$ used CBCT to evaluate dehiscence and fenestration in patients with class I and class II division 1 malocclusions. In their results, fenestration was observed in $36.5 \%$ of the samples. Of these fenestrations, $46.6 \%$ were found in the maxillary anterior teeth and $68.8 \%$ in the mandibular anterior teeth.

Orthodontic treatment for adults has become increasingly common. In a study comparing the thickness of the lower anterior alveolar bone in the mixed dentition, early permanent dentition, and adult dentition, Choe et al. ${ }^{14}$ found that the thickness of buccal and lingual alveolar bone tended to decrease from adolescence to adulthood. In addition, adult patients are more likely to develop periodontal support deficiencies due to a decrease of periodontal ligament activa- 
tion, plaque accumulation, the onset of periodontal disease, and alveolar bone loss. Additionally, the amount of compensatory bone formation decreases with aging, so tooth movement can be limited based on the alveolar housing. Careful treatment planning must include assessment of the status of the bone housing the teeth to be moved.

If no skeletal discrepancy is present, orthodontic treatment that requires substantial tooth movement may be advantageous when performed during a period of active growth. However, orthodontic treatments in adult skeletal class III patients are often performed at the end of growth, and in such cases, the alveolar bone thickness should be considered comprehensively. Kim et al. ${ }^{15}$ found that adult patients with class III malocclusion and high angles often have thin alveolar bone in the lower anterior region, and they suggested that treatment planning should take into account the limitations on tooth movement that the alveolar bone may present.

A limitation common to all studies that use CBCT for measurements is the spatial resolution of CBCT. Important factors influencing spatial resolution are partial volume averaging and scattering radiation. ${ }^{16}$ Thin, delicate structures, such as the buccal or lingual alveolar bone, the temporal bone, and the sinus wall of the sphenoid, are especially susceptible to the partial volume effect. Scatter radiation in CBCT increases as the size of the FOV increases. The larger the FOV, the lower the resolution of the image. This can make it very difficult to detect thin bone $(<1 \mathrm{~mm})$. The appropriate way to prevent poor resolution is to use the smallest FOV that encompasses the region of interest. ${ }^{17}$

The results of the present study showed that moving the mandibular incisors too far forward during preoperative orthodontic treatment could lead to alveolar bone loss around the mandibular incisors. Therefore, it is important to pay special attention to patients with mandibular prognathism.

\section{Conflicts of Interest: None}

\section{References}

1. Wennström JL. Mucogingival considerations in orthodontic treatment. Semin Orthod 1996; 2: 46-54.

2. Handelman CS. The anterior alveolus: its importance in limiting orthodontic treatment and its influence on the occurrence of iatrogenic sequelae. Angle Orthod 1996; 66: 95-110.
3. Wehrbein H, Bauer W, Diedrich P. Mandibular incisors, alveolar bone, and symphysis after orthodontic treatment. A retrospective study. Am J Orthod Dentofacial Orthop 1996; 110: 239-46.

4. Vardimon AD, Oren E, Ben-Bassat Y. Cortical bone remodeling/tooth movement ratio during maxillary incisor retraction with tip versus torque movements. Am J Orthod Dentofacial Orthop 1998; 114: 520-9.

5. Kim Y, Park JU, Kook YA. Alveolar bone loss around incisors in surgical skeletal Class III patients. Angle Orthod 2009; 79: 676-82.

6. Nahm KY, Kang JH, Moon SC, Choi YS, Kook YA, Kim SH, et al. Alveolar bone loss around incisors in Class I bidentoalveolar protrusion patients: a retrospective three-dimensional cone beam CT study. Dentomaxillofac Radiol 2012; 41: 481-8.

7. Nelson PA, Artun J. Alveolar bone loss of maxillary anterior teeth in adult orthodontic patients. Am J Orthod Dentofacial Orthop 1997; 111: 328-34.

8. Janson G, Bombonatti R, Brandão AG, Henriques JF, de Freitas MR. Comparative radiographic evaluation of the alveolar bone crest after orthodontic treatment. Am J Orthod Dentofacial Orthop 2003; 124: 157-64.

9. Baumrind S, Frantz RC. The reliability of head film measurements. 2. Conventional angular and linear measures. Am J Orthod 1971; 60: 505-17.

10. Nakajima K, Yamaguchi T, Maki K. Surgical orthodontic treatment for a patient with advanced periodontal disease: evaluation with electromyography and 3-dimensional conebeam computed tomography. Am J Orthod Dentofacial Orthop 2009; 136: 450-9.

11. Lee SL, Kim HJ, Son MK, Chung CH. Anthropometric analysis of maxillary anterior buccal bone of Korean adults using conebeam CT. J Adv Prosthodont 2010; 2: 92-6.

12. Yagci A, Veli I, Uysal T, Ucar FI, Ozer T, Enhos S. Dehiscence and fenestration in skeletal Class I, II, and III malocclusions assessed with cone-beam computed tomography. Angle Orthod 2012; 82: 67-74.

13. Evangelista K, Vasconcelos Kde F, Bumann A, Hirsch E, Nitka M, Silva MA. Dehiscence and fenestration in patients with Class I and Class II Division 1 malocclusion assessed with cone-beam computed tomography. Am J Orthod Dentofacial Orthop 2010; 138: 133.e1-7.

14. Choe HY, Park W, Jeon JK, Kim YH, Shon BW. Differences in mandibular anterior alveolar bone thickness according to age in a normal skeletal group. Korean J Orthod 2007; 37: 220-30.

15. Kim YS, Cha JY, Yu HS, Hwang CJ. Comparison of mandibular anterior alveolar bone thickness in different facial skeletal types. Korean J Orthod 2010; 40: 314-24.

16. Chakeres DW. Clinical significance of partial volume averaging of the temporal bone. AJNR Am J Neuroradiol 1984; 5: 297302 .

17. Miracle AC, Mukherji SK. Conebeam CT of the head and neck, part 1: physical principles. AJNR Am J Neuroradiol 2009; 30: 1088-95. 